\title{
Online, Health-Related Discussion Groups
}

\author{
What We Should Know and Do
}

$I^{n}$ $\mathrm{n}$ this issue, Culver and colleagues analyze messages on an Internet-based electronic bulletin board. ${ }^{1}$ They conclude that the medical information on this bulletin board may be inaccurate, inappropriate, and based on limited evidence. Their results raise issues about the impact of such information on internists and patients. What are we to make of these results? First, we must appreciate that the Internet is affecting information exchange more profoundly and rapidly than perhaps any other innovation in history. It provides patients, physicians, and others with unprecedented opportunities to learn. inform. and communicate with one another. One study found that more than $37 \%$ of America's "wired" households regularly seek online medical information. (J. Schwartz. Washington Post. April 22. 1997. Health Suppl.). The U.S. government's Internet site for helping consumers find high-quality health information was visited 4.8 million times in its first 30 days of operation. according to its press release (http://www.healthfinder.gov; see the "about" link). The Internet is becoming an important new source of health information.

One of the more interesting aspects of the Internet phenomenon is that groups of people from around the globe can share ideas in a rapid. convenient. and inexpensive way. An amount of text equivalent to 800 novels. each with 400 pages, is posted each day to one system of Internet discussion groups. and this volume has doubled each year for the last few years (http://www.dejanews. com/help/dnusenet_help.html\#size: help document on the Dejanews web site which gives volume of daily postings to Usenet newsgroups). Online forums like the bulletin board discussed in this issue are an increasingly important resource for physicians and patients seeking education. gutdance in clinical decision making. and interpersonal connections.

Groups of individuals use two main methods to conduct such interactions: listservers. which also are known as mailing lists. and newsgroups. Both methods allow people to write messages that can be read and answered by all other members of the group. ${ }^{2}$ There are dozens of healthrelated mailing lists and newsgroups. Although precise statistics are difficult to track. the popularity of these forums is growing because less-expensive Internet access and more intuitive Web browsers have made them more accessible.

These self-help groups can provide patients with useful benefits. They give patients access to coping strategies and the emotional support that clinicians might not have the time or personal experience to offer. Patients share practical advice and encourage one another to deal with the daily challenges faced by those with chronic disease. Many patients describe a sense of fulfilment as they evolve from needy support seekers to seasoned advice givers for others in need. These groups provide useful infor- mation, sympathy, validation, and personal mastery, which also may help explain their growing popularity.

Online discussions also have less-desirable features. Like all social interactions among diverse individuals. these discussions can be marred by lengthy and inconsequential chatter. rudeness, and domination by a few participants. The study by Culver and colleagues focuses attention on more serious problems. including misinformation and the reinforcement of disability. Unfortunately. it is often difficult for users to assess the quality of information. When launching the U.S. Government's "healthfinder" web site. "[Vice President] Gore called the wealth of online consumer health information a 'mixed blessing.' because 'finding high-quality information that is accurate. timely. relevant and unbiased is a daunting challenge to even the most experlenced Web surfer" (J. Schwartz, Washington Post. April 22. 1997. Health Suppl.). Help is becoming available. The "healthfinder" web site is an example of a quality filter for such information. Also, formal and informal codes of conduct for the exchange of online health information (see HONCode. Health on the Net Foundation. http: //www.hon.ch) are beginning to emerge. "Their basic principle is that users should be able to determine who is offering information and why.

There is another result in the study by Culver and colleagues that should concern us. A significant percentage of the messages contained complaints about medical care. This observation suggests that one of the many reasons patients turn to faceless strangers in these discussion groups is that they are not getting important needs filled by their physicians. We must ask ourselves how we can address these needs better. To the extent that unmet needs remain. we should consider whether guiding appropriate patients with specific problems to these support groups might supplement the care we provide.

Our decision to guide patients to online discussion groups should be based on evidence about their benefits and hazards. Unfortunately. the literature on this topic is scant. For example, a MEDLINE search using the $\mathrm{MeSH}$ heading "self-help groups" and either the MeSH heading "computer communication networks" or the text word "newsgroups" yielded only a few articles. Even when taken together. these articles do not provide the information needed to decide.

The study by Culver and colleagues provides information about one discussion group devoted to one problem. Its greater importance is that it helps define the methodologies that can provide more information. We need further analyses along similar lines across the broad range of health-related discussion groups to clarify their substance, dynamics, harms, and benefits. If these studies show that some discussion groups are useful adjuncts to traditional care, another generation of studies will be 
needed to define optimal strategies for incorporating these resources into our therapeutic tool kit.

While we wait for further data, we should consider reading the messages in some Internet discussion groups ourselves. Doing this will make us more comfortable asking patients if they use these resources and enable us to offer thoughtful counsel about them. The history of alternative medicine has taught us that patients use nontraditional health care resources, frequently without our participation or knowledge. ${ }^{4}$

The Internet and World Wide Web offer much more than online support groups. There are forums solely for clinicians: for example. only American College of Physician (ACP) members can participate in clinical discussions on the ACP web site (http://www.acponline.org). There is information for physicians, patients, or both. such as the U.S. government's web site. cited above. and there are "electronic house calls" provided by physicians and computer-based expert systems. ${ }^{E}$ Creative investigators are even using Intemet discussion groups to gather research data. ${ }^{6}$

Some web sites make it easier to find health-related discussion groups and other useful medical information on the Internet. Liszt (www.liszt.com) and Dejanews (www. dejanews.coml are examples of sites whose primary purpose is to provide links to newsgroups and listservers. including those devoted to health and medicine. So-called medical supersites provide one-stop shopping for links to a broad range of medical information on the Internet. For example. the U.S. Government's "healthfinder" web site was designed to make it easier to find reliable health information on the World Wide Web. This site covers a wide range of health care topics addressed by the federal govemment and its many partners and includes links to many health-related discussion groups. Online tours provide examples of the disease-specific resources and support available through this site. An important feature of such sites is the creative use of public and private sector collaborations to ensure widespread dissemination to all socioeconomic groups. ${ }^{\bar{x}}$ Therefore, whatever one's opinion about patients' use of the Internet, substantial forces are at work to make online health care resources available to them.

Many nongovernmental supersites also are available. A long-standing and frequently referenced example is Medical Matrix (www.medmatrix.org). which according to its home page, provides "ranked, peer-reviewed, annotated. updated clinical medicine resources." Users can identify sites in a variety of categories. such as news. journals, forums, continuing medical education programs. patient education programs. specialty, and disease. $A$ variety of discussion groups are covered, and any of the hundreds of sites can be visited by clicking the corresponding link. Other supersites are provided by pharmaceutical companies, individual physicians, medical societies, uni- versities. publishers, and others. When examining information in these sites, it is essential to determine who is posting, selecting, or commenting on the material, including their qualifications. selection methods. and underlying motivations.

There are a variety of strategies for learning more about the Internet's discussion groups and other medically related information. Try visiting several supersites and browsing through their offerings. Consider picking a clinical topic that plays an important role in your practice. Diabetes and cancer are examples of common diseases with popular online discussion groups. Alternatively. investigate discussion groups concerned with less-common diseases such as albinism and multiple sclerosis. In your travels you will most likely find other useful information including patient handouts. guidelines. teaching modules. and evidence-based medicine resources. Some people suggest that physictans and patients explore the Internet together. This practice could help you teach patients to interpret online information and refine your understanding of how this information can best meet their needs. Given that the Internet is becoming an increasingly important resource for us and our patients. time spent mastering it is wisely invested.-Jerome A. OsherofF, MD, Clinical Information Management Department. American College of Physicians, Philadelphia, Pa.

\section{REFERENCES}

1. Culver JD, Gerr F, Frumkin H. Medical information on the Internet: a study of an electronic bulletin board. J Gen Intern Med. 1997:12:466-70.

2. Peters R, Sikorski R. Sharing information and interests on the Intemet. JAMA. 1997:277:1258.

3. Silberg WM. Lundberg GD. Musachio RA. Assessing. controlling and assuring the quality of medical information on the Internet: Caveat Lector et Viewor-Let the reader and viewer beware. JAMA. 1997:277:1244 5.

4. Eisenberg DM. Kessler RC. Foster C. Unconventional medicine in the United States: prevalence, costs, and patterns of use. $\mathrm{N}$ Engl $\mathrm{J}$ Med. 1993:328:246-52.

5. Engstrom P. Brown MS. Electronic house calls: new rules, new roles as healers swarm the net. Medicine on the Net. Newsletter December 1996:2(12). Cor Health Resources (see http://www.mednet-i. com for ordering information].

6. Engstrom P. How a scientist tapped UseNet for clues to an inexplicable disease. Medicine on the Net. Newsletter March 1997:313). Cor Health Resources (see http:/www.mednet-i.com for ordering information).

7. Engstrom P. Wide connections: is online healtheare for the privileged few? NetWellness thinks not-and hopes to prove its case. Medicine on the Net. Newsletter May 1997:3[5]. Cor Health Resources (see http://www.mednet-i.com for ordering information. and http:// www.netwellness,org for Netwellness).

8. Lefebvre A. Self care online: a doctor's best friend. Medicine on the Net. Newsletter May 1997:3(5). Cor Health Resources (see http:// www.mednet-i.com for ordering information). 